\title{
Design of the combinational logic circuit system for automatic control suction pump
}

\author{
Minghui You ${ }^{1}$, Qixiang Sun ${ }^{1}$, Liping Yin ${ }^{1}$, Shijun $\mathrm{LI}^{1}$, Xue $\mathrm{Li}^{1}$, Jingsheng $\mathrm{Liu}^{1, \text { a }}$ \\ ${ }^{1}$ Jilin Agricultural University, Changchun 130118, Jilin, China \\ amhyou000@163.com
}

Keywords: combinational logic circuit ,automatic control,circuit design,suction pump

\begin{abstract}
In this paper, we analysis and designed an automatic control circuit, which is very important for vehicle permitted both automatically controlled and a single LED ,respectively. The results showed the functions that The suction pump works properly can be adjusted with automatic control circuit. An automatic control system that is controlled by one or more single - board computers. This upaper introduces the design process of combinational logic circuit , and the working principle of the system.
\end{abstract}

\section{Introduction}

Automatic control system of suction pump is practical use whatever. [1]With the development of our economy, urban and rural residents living standards has improved. Many families have installed a water tower pumping water. [2] When manual control pumping process, the operation is the intricacy of the work. [3] An automatic control system are key parts that protect suction pump from damage. [4]The combinational logic circuit has the advantages of simple structure, reliable operation, low cost and other characteristics.[5]

The system devices can perform a variety of control functions in electronic equipment. Design of combinational logic circuits is important.Determine the input and output. and then according to the output and the input of the logical relationship between the list of true value table. [6]The selection of device types. According to the requirements of decision and device functions. [7]For example, when the circuit design of MSI devices, for multi output functions, the decoder is convenient, and the single output function, then use the data selector is convenient. [8]According to the true value table type and selection logic device, write logic function expression. When using SSI integrated gate design, the logic function simplification and transformation as the most simple gate circuit corresponding. [9]Draw the logic circuit diagram according to the logic function expression and the selection of logic device.

\section{Overall System Framework}

\section{1 operating principle}

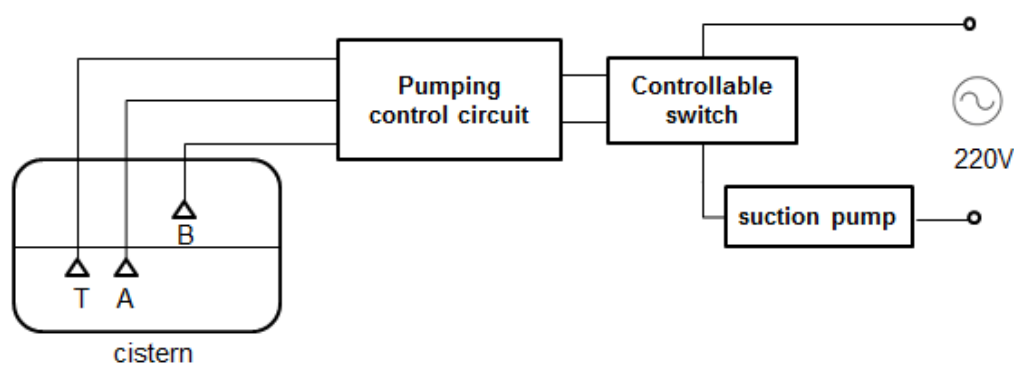

Fig. 1 Automatic control diagram of suction pump

\subsection{Determine the logical function:}

In many practical problems ,the circuit which the schematic was designed was used to determined the logic function . 
Common output and input, output or another output to indicate there lationship between. Three detectors were placed in the cistern, the detection results as input variables, respectively A, B, T.And using controllable switch. The output variable or disconnected. Through the establishment of automatic controllable circuit, makes the whole circuit capable of automatic water supply when the level of water was lower.

\subsection{Working Process}

The first step to system modeling and control was input selection. When there was no water in the cistern,the circuit of three probes were not conduction. When A and B input was low, the output was high. Sensors and automatic control technology was an important link in achievingtest.

Control switch started pumping motor with only the water level . When the water level was both higher than $\mathrm{A}$ and lower than $\mathrm{B}$, the circuit of $\mathrm{A}$ and $\mathrm{T}$ conducted.

The principle is if the sensor pumping, the output high, go on with its work. When no pumping, the output low,had a rest.When the level was higher than B, the circuit system brought the pump to a rest. A single yellow LED1 shows that the power was switched on. The green LED2 shows there was water. And the red LED3 shows there was no water.

\section{Circuit Design}

The combinational logic circuit has the advantages of simple structure, reliable operation, low cost and other characteristics. Therefore, the electronic devices are widely applied in small and medium scale. For example: encoder, decoder, data selector and so on. According to the logic function , the process design of combinational logic circuit, draw the corresponding logic circuit. The working process of the front has a clear logic function circuit, and the input and output variables, we proceed to the next step, according to the logic function requirements, lists the truth table.

Table 1 Function Table

Table 2 Truth Table

\begin{tabular}{cccccccc}
\hline Probe A & Probe B & $\mathrm{Q}_{\mathrm{n}}$ & $\mathrm{Q}_{\mathrm{n}+1}$ & $\mathrm{~A}$ & $\mathrm{~B}$ & $\mathrm{Q}_{\mathrm{n}}$ & $\mathrm{Q}_{\mathrm{n}+1}$ \\
\hline No water & No water & No pumping & Pump & 0 & 0 & 0 & 1 \\
No water & No water & Pump & Pump & 0 & 0 & 1 & 1 \\
Water & No water & Pump & Pump & 1 & 0 & 1 & 1 \\
Water & No water & No pumping & No pumping & 1 & 0 & 0 & 0 \\
Water & Water & No pumping & No pumping & 1 & 1 & 0 & 0 \\
Water & Water & Pump & No pumping & 1 & 1 & 1 & 0 \\
No water & Water & - & - & 0 & 1 & - & - \\
\hline
\end{tabular}

The logical expression:

$$
\begin{aligned}
& \vartheta_{n+1}=\bar{A} \bar{B} \bar{Q}_{n}+\bar{A} \bar{B} \vartheta_{n}+A \bar{B} \vartheta_{n} \\
& =\overline{A B}\left(\overline{\theta_{n}}+Q_{n}\right)+A \bar{B} Q_{n} \\
& =\overline{A B}+A \bar{B} Q_{n} \\
& =\overline{A B}+A \bar{B} Q_{a} \\
& \vartheta_{a+1}=\bar{B} \overline{A \overline{\theta_{a}}}
\end{aligned}
$$

According to the logical expression, draw the logic diagram 


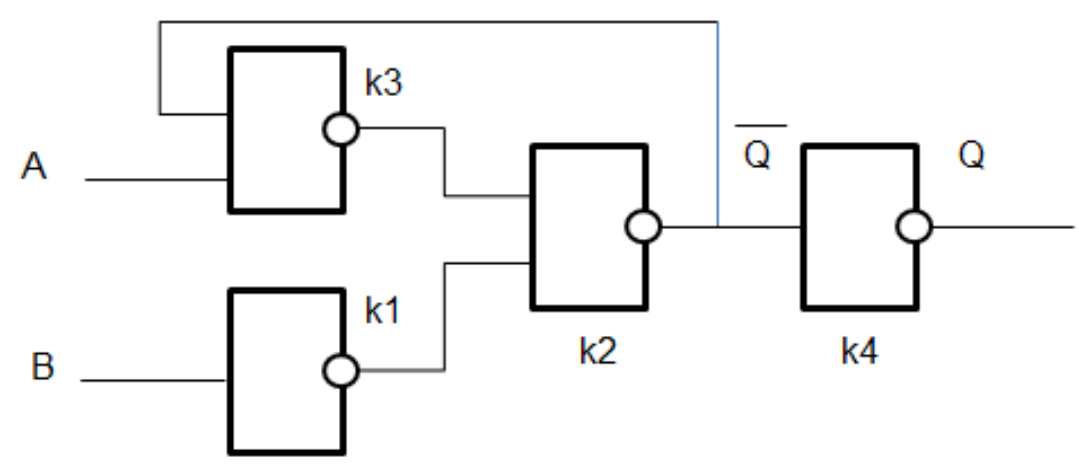

Fig. 2 Logic circuit diagram

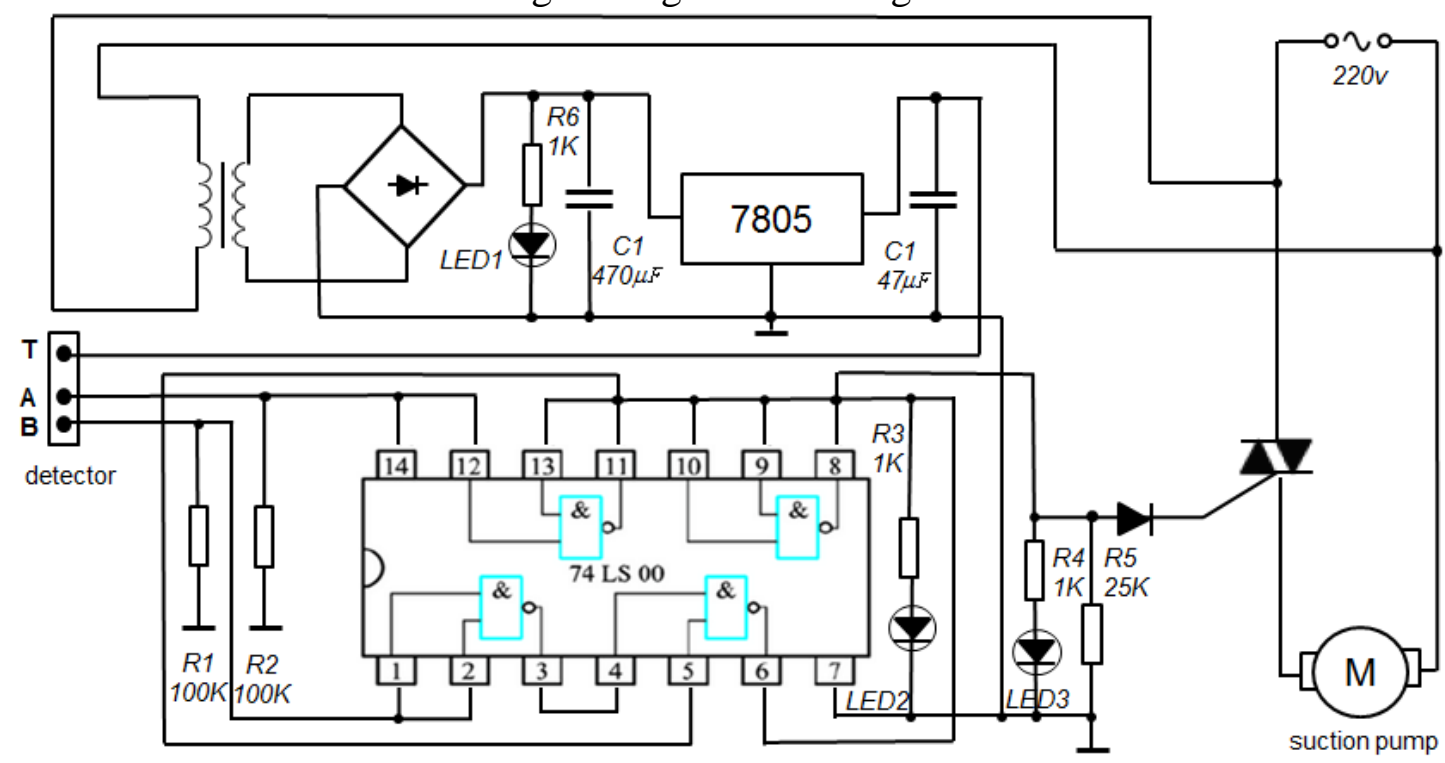

Fig. 3 Principle of operation

According to the verification of the actual circuit, the design of the control circuit is able to complete its logic function.

\section{Summary}

The main function of the system is to automatically detect the water level and pumping function. When the water level is too low, circuit, water pump automatic pumping; when the water level is too high, the circuit breaker, the pump automatically stops pumping. In order to facilitate the observation, each functional circuit series LED. system with different colors through a combination of logic circuits to realize the automatic control function.

\section{Acknowledgments}

The authors wish to express their gratitude to the projects: Jilin Province Education Department Project (No. 2015Y174), Jilin Agricultural University learning program, Jilin Provincial Department of human resources and social security Project (No. 2015Y24)for their generous support of this work.

\section{References}

[1]Z Gan. Automatic Synthesis of Combinational Logic Circuit with Gene Expression-Based Clonal Selection. [J] Algorithm International Conference on Natural Computation, 2008, 6:278-282 
[2] AM Wahba. A method for automatic design error location and correction in combinational logic circuits. [J] 《Journal of Electronic Testing》, 1996, 8(2):113-127

[3] Cheng H. The Design of the Parasol in Automatic Control System Based on the Logic Circuits. [J]Journal of Shangqiu Vocational \& Technical College, 2011.

[4] Nagai S, Shiomi H. Automatic control system: US, [P] US 6405099 B1. 2002.

[5] Soccoli P, Ostrovsky M, Lombardi A J. Lighting control system \& three Way occupancy sensor: US, [P]US20070216313. 2007.

[6] Guldner J, Sienel W, Tan H S, et al. Robust automatic steering control for look-down reference systems with front and rear sensors. [C] IEEE Transactions on Control Systems Technology, 1999, 7(1):2-11.

[7] Matiur Rahman S A M, Al Mamun M A, Ahamed N U, et al. Design of automatic controlling system for tap-water using floatless level sensor// [C]IEEE International Symposium on Robotics and Manufacturing Automation. IEEE, 2014.

[8] Yang H M, Kong W C, Yue L I, et al. Based on the twist ring counter design traffic light automatic control circuit. [J].Electronic Design Engineering, 2013.

[9] LI Ai-jun1, SHEN yi2, ZHANG Wei-guo1 The Design of Fuzzy Control System for Aircraft Automatic Landing; [J]Ordnace Industry Automation;2002-06. 